\title{
Design and study of PEG Linkers that Enable Robust Characterization of PEGylated Proteins
}

Anumita Saha-Shah ${ }^{1 *}$, Shuwen Sun ${ }^{2}$, John Kong ${ }^{3}$, Wendy Zhong ${ }^{3}$, Benjamin F. Mann ${ }^{3}$

${ }_{1}$ Process Research and Develoment, Merck \& Co.Inc. 770 Sumneytown Pike, West Point, Pennsylvania 19486, USA

2 Process Research and Develoment, Merck \& Co.Inc. 2000 Galloping Hill Road, Kenilworth, New Jersey 07033, USA

${ }^{3}$ Process Research and Develoment, Merck \& Co.Inc. 126 E. Lincoln Ave, Rahway, New Jersey 07065, USA

\section{Table of Content:}

Schematic of Transglutaminase catalysis $\ldots \ldots \ldots \ldots \ldots \ldots \ldots \ldots \ldots \ldots \ldots \ldots \ldots \ldots \ldots . .2$

Deconvoluted intact mass spectra of IFN and IFN-PEG(10) conjugates................. S-3

IFN-PEG(5K) conjugate sequence coverage map..................................

Detailed characterization data (MS1 and MS2 spectra) for K134 and K31 modification.....S-5

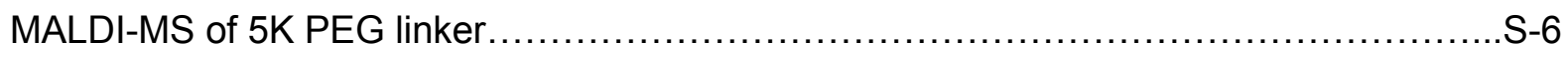

Size exclusion-multiangle light scattering results of IFN-5K PEG conjugate $\ldots \ldots \ldots \ldots \ldots \ldots$. -7 
Transglutaminase catalysis:<smiles>CN[C@@H](CCC(=O)SCC1CCCCCCC1)C(C)=O</smiles><smiles>CN[C@@H](CCCCNC(=O)CC[C@H](NC)C(C)=O)C(C)=O</smiles>

Figure S1: Schematic showing mechanism of transglutaminase catalysis 


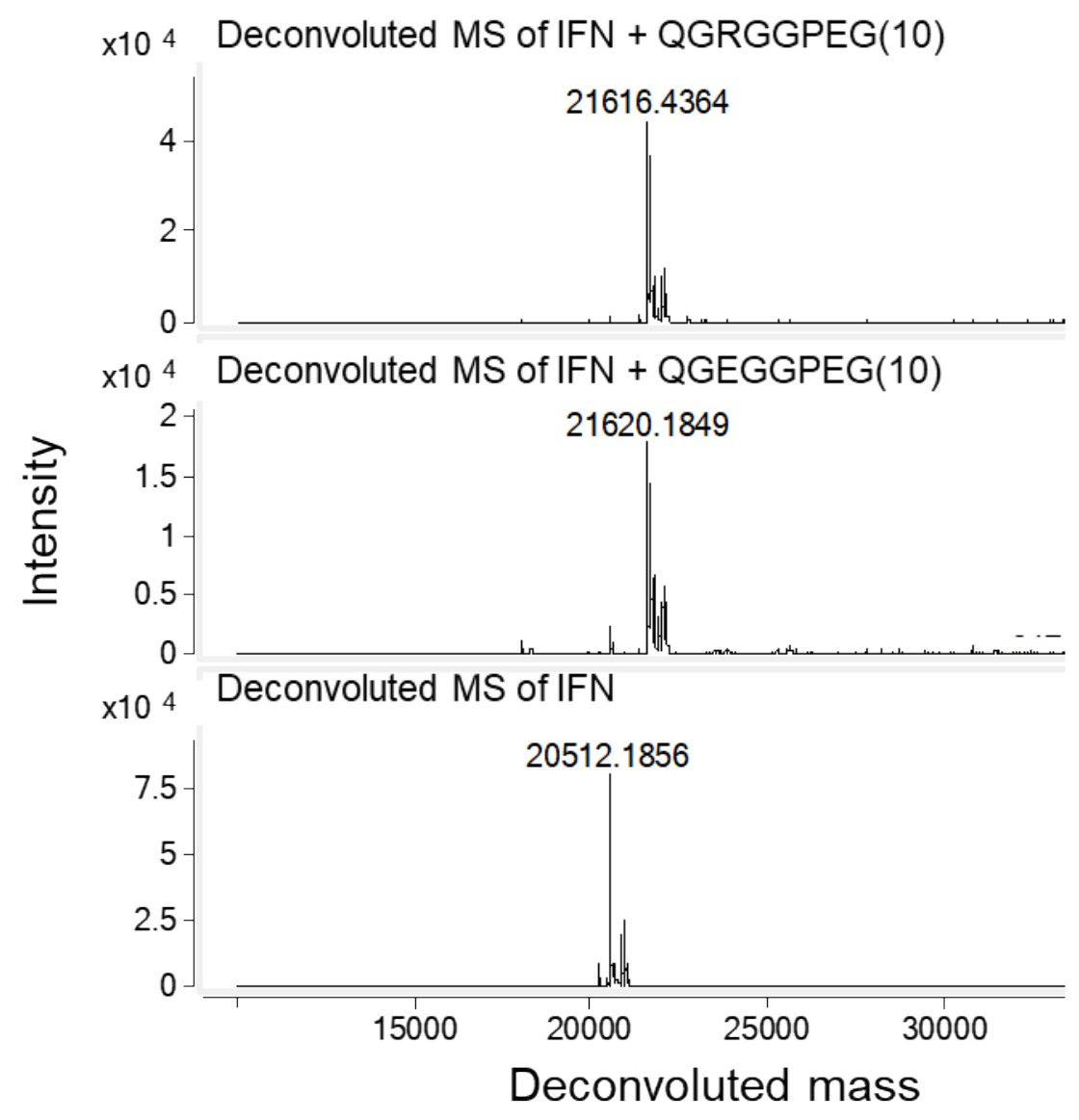

Figure S2: Deconvoluted intact mass spectra of IFN and IFN-PEG(10) conjugates 


\section{IFN-PEG(5K) conjugate sequence coverage map:}

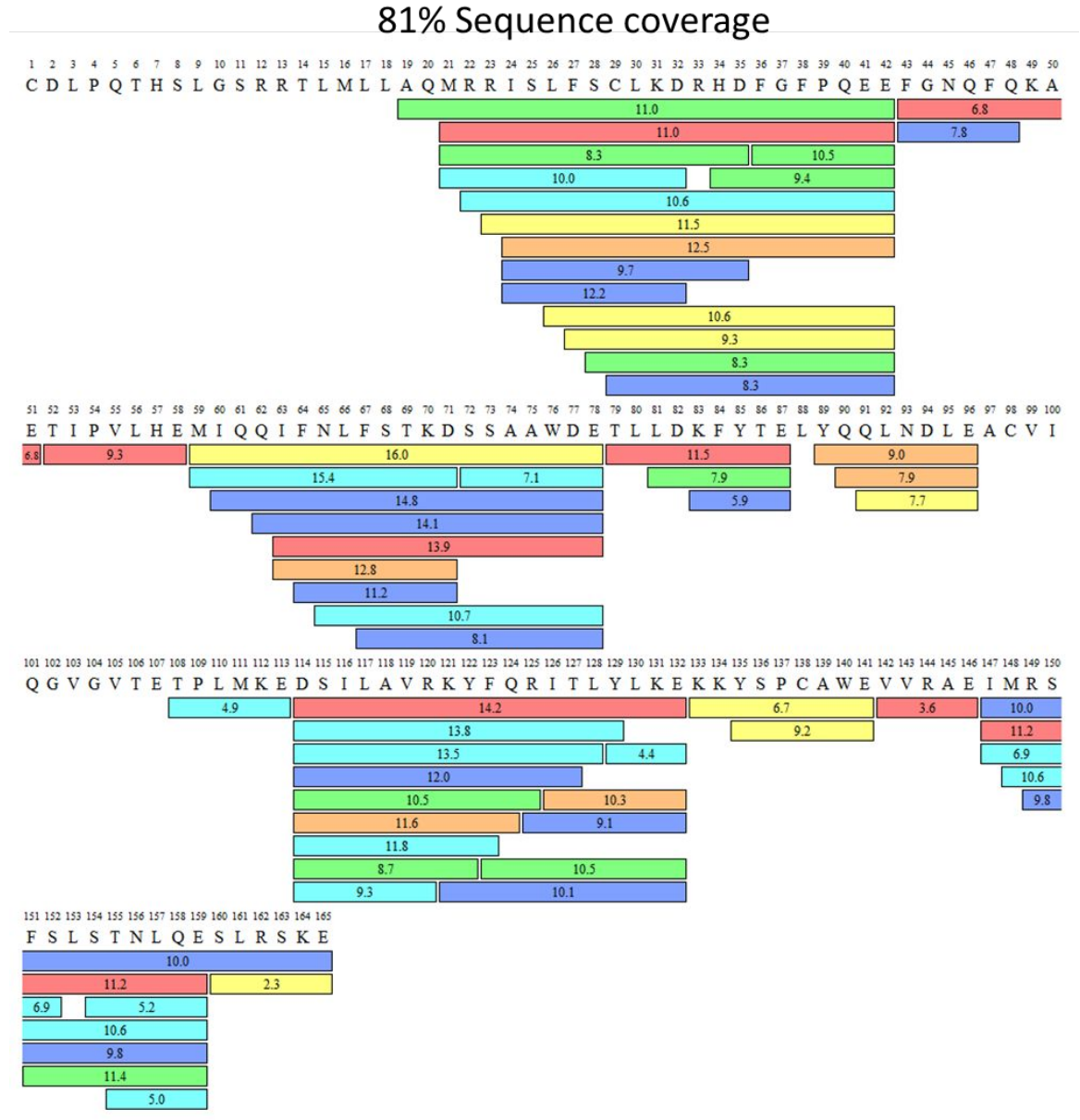

Figure S3: Peptide map showing sequence coverage of IFN-PEG(5K) conjugate post- GluC digestion 
MS1 and MS2 data showing K134 and K31 modification:

\section{K31 modification analysis}

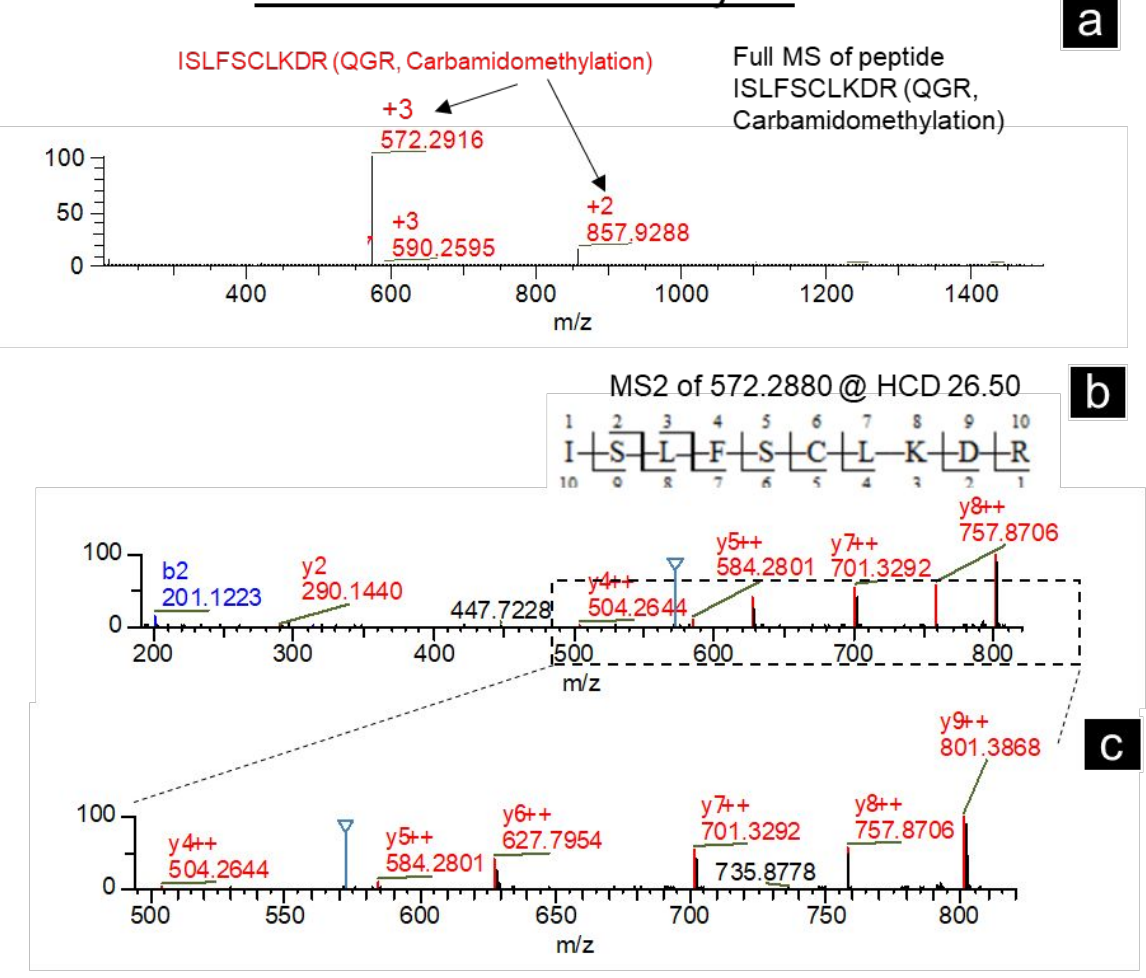

$\underline{\text { K134 modification analysis }}$

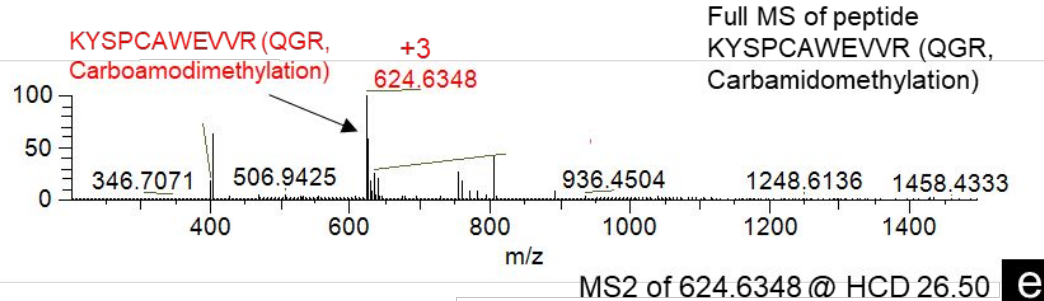

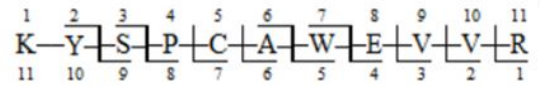

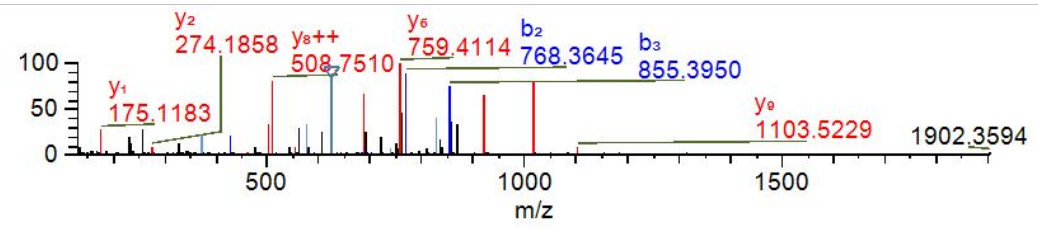

Figure S4: MS1 (a) and MS2 (b) spectra of peptide ISLFSCLKDR indicating QGR modification at K31, a zoomed-in spectra of b showing all b-ions is depicted in c. MS1 (d) and MS2 (e) spectra of peptide KYSPCAWEVVR indicating QGR modification at K134 
MALDI-MS of Q(cbz)GRGG-PEG(5K):

MALDI-MS data of the 5K PEGs obtained from GenScript were performed for quality control using DHAP martix and methods detailed in the experimental section of the manuscript.

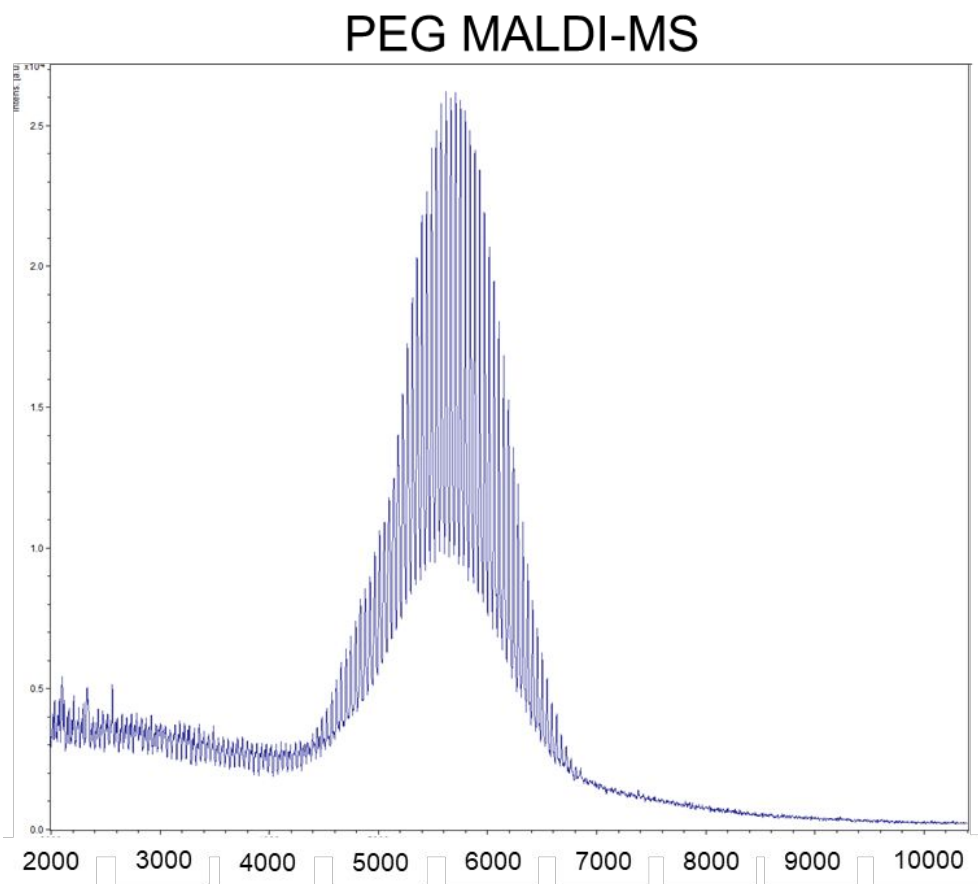

Figure S5: MALDI-MS of QGRGG-PEG(5K)

\section{Size exclusion-multiangle light scattering:}

Agilent 1200 HPLC connected to Wyatt Dawn multiangle light scattering, UV (280 nm, Agilent) and refractive index (Wyatt) detector was used for this study. A TSKgel G3000SWXL, $7.8 \mathrm{~mm} * 300 \mathrm{~mm}$, 5um column at room temperature and $150 \mathrm{mM}$ PBS $(\mathrm{pH} 7.4)$ mobile phase was used for size exclusion chromatography. Isocratic flow of PBS buffer at $0.5 \mathrm{~mL} / \mathrm{min}$ for $26 \mathrm{~min}$ was used. Bovine serum albumin (100 ug per injection) was used to test system suitability before any injection. $100 \mathrm{ug}$ of conjugated IFN was injected for each run, concentration was measured from refractive index detector. A dn/dc of 0.185 which is typical of protein was used for calculations. Dn/dc of PEG was calculated to be 0.13 . 


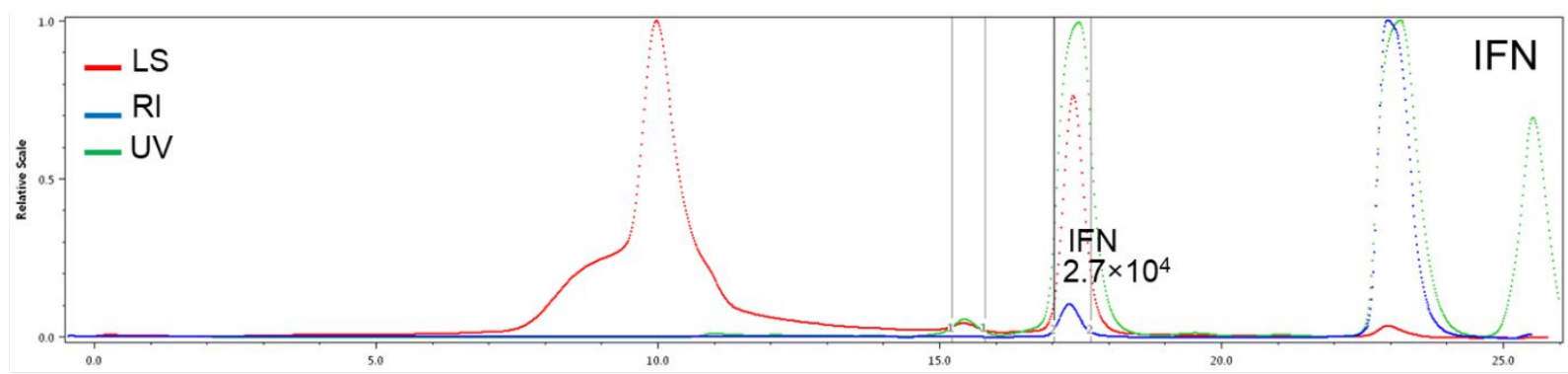

IFN-PEG (5k) conjugate

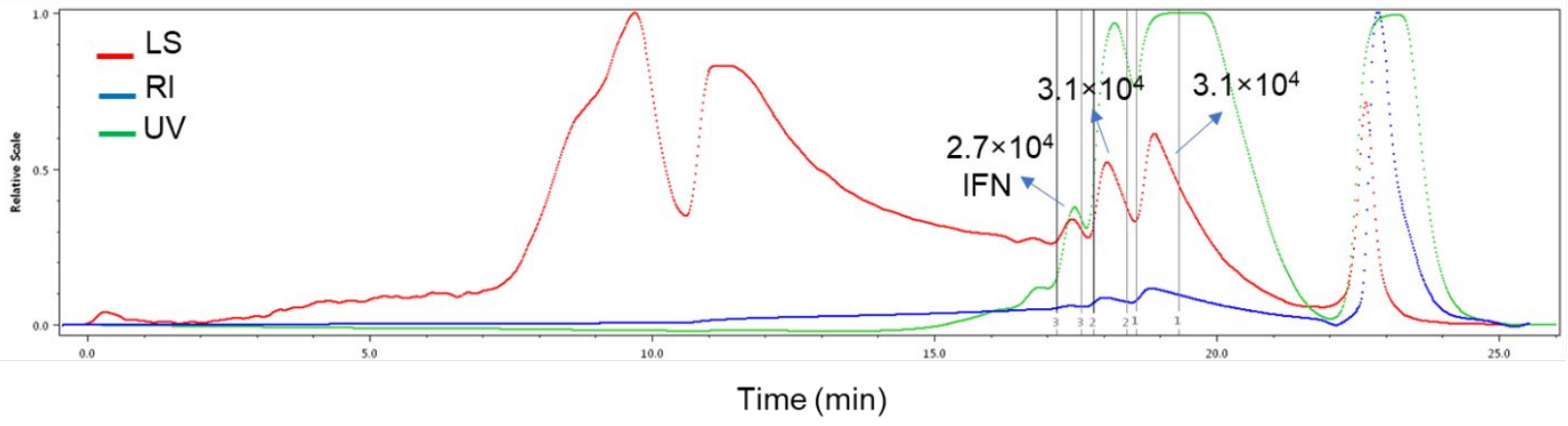

Figure S6: SEC-MALS data also indicates formation if IFN-QGRGG-PEG(5K) conjugate 